\section{Group formation and cohesion of active particles with visual perception-dependent motility}

\author{
François A. Lavergne, Hugo Wendehenne, Tobias Bäuerle, Clemens Bechinger*
}

Group formation in living systems typically results from a delicate balance of repulsive, aligning, and attractive interactions. We found that a mere motility change of the individuals in response to the visual perception of their peers induces group formation and cohesion. We tested this principle in a real system of active particles whose motilities are controlled by an external feedback loop. For narrow fields of view, individuals gathered into cohesive nonpolarized groups without requiring active reorientations. For wider fields of view, cohesion could be achieved by lowering the response threshold. We expect this motility-induced cohesion mechanism to be relevant not only for the self-organization of living systems, but also for the design of robust and scalable autonomous systems.

@ roups of individuals can organize into an abundant diversity of spatiotemporal patterns, such as flocks of birds (I), schools of fish (2), or human crowds (3). This complex collective behavior results from the dynamical response of individuals to visual (4), acoustic (5), or chemical (6) stimuli perceived from their peers. Depending on their perception, individuals change their orientation (alignment), nearest-neighbor distance, or velocity, which leads to different states such as disordered swarms, directed flocks, or rotating swirls (7). As a result of variations of the individuals' behavior, groups can dynamically switch between states while still remaining cohesive (8), which suggests the presence of robust cohesion mechanisms.
The recent development of synthetic systems of active Brownian particles (ABPs), known as active matter, has been used as a bottom-up approach to identify the minimal ingredients needed to reproduce natural collective phenomena $(9,10)$. For instance, ABPs were demonstrated to form densely packed clusters (11-13), even for purely repulsive interacting systems (14). Nonetheless, these clusters are not intrinsically cohesive but only appear in coexistence with a dilute background of ABPs (15). In the presence of velocity alignment, large flocks with directional motion have been obtained $(16,17)$. However, to obtain truly cohesive groups within entirely empty surroundings, as observed in nature, one needs the addition of pairwise attractions (18) or active reorientations $(19,20)$. Here, we used an experimental system based on an external feedback (21), where ABPs individually vary their velocity depending on the visual perception of their peers. We demonstrate that this is sufficient to obtain a single cohesive nonpolarized group from any number of particles, without the need for pair attraction or active reorientation. Not only is our experimental realization valid in idealized systems, it also confirms the robustness of this cohesion mechanism to the inherent noise and imperfections of a real system.

We first introduce our measure of perception as a simplified vision scheme. As illustrated in Fig. 1A, an individual $i$ with orientation $\hat{p}$ can see other individuals $j$ only within a restricted vision cone of half angle $\alpha$, denoted $V_{i}^{\alpha}(22,23)$. We quantify this visual stimulus by the perception function

$$
P_{i}=\sum_{j \in V_{i}^{a}} \frac{1}{2 \pi r_{i j}}
$$

where $r_{i j}$ is the distance between $i$ and $j$. Note that our choice of a metric perception, where the signal decays with distance, is motivated by its established role in the swarming of insects (24). However, our aim is not to model vision, but rather to investigate the presence of a simple and generic long-range perception mechanism whose phenomenology may not be restricted to our experimental system. Crucially for $\alpha<\pi$, the vision cone makes perception anisotropic, which leads

Department of Physics, University of Konstanz, 78464 Konstanz, Germany.

*Corresponding author. Email: clemens.bechinger@ uni-konstanz.de
A

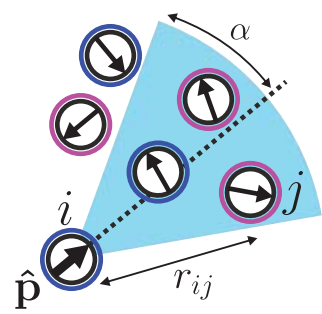

B

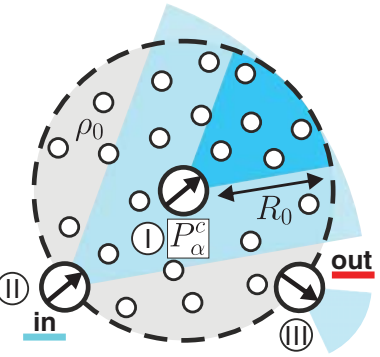

D

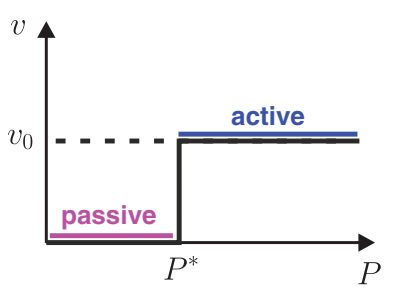

C

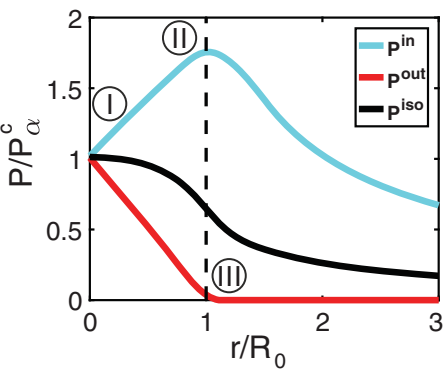

E

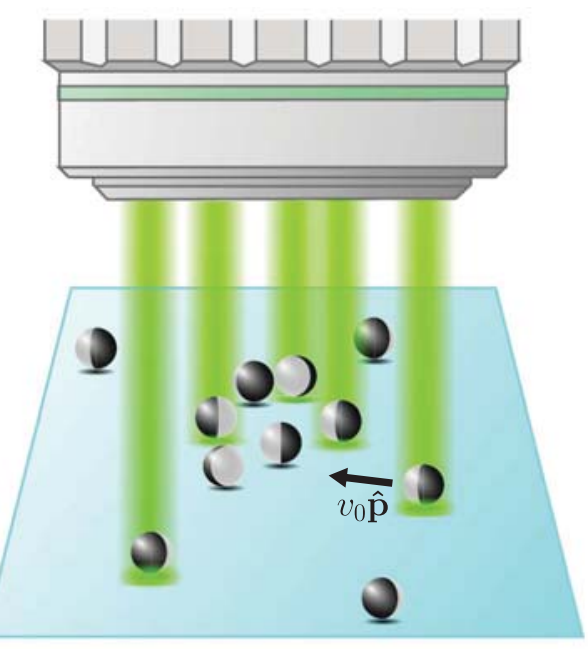

Fig. 1. Active particles with visual perception-dependent motility.

(A) Perception of an individual through a vision cone of half angle $\alpha$. See (D) for the blue/magenta color code. (B) Perception of a circular group of individuals for three different observers: at the center $\left(P=P_{\alpha}^{\mathrm{c}}, \mathrm{I}\right)$ and at the edge pointing inward (II) or outward (III). (C) Numerically computed perception profiles $P^{\text {in }}$ (cyan) and $P^{\text {out }}$ (red) for the group in (B) with $\alpha=\pi / 4$, and $P^{\text {iso }}$ corresponding to isotropic vision ( $\alpha=\pi$, black). Cases I, II, and III of (B) are reported on the graph. (D) Motility response to the visual perception. Above a threshold $P^{*}$, particles are active with $v=v_{0}$ and are colored blue; below that threshold, particles are passive with $v=0$ and are colored magenta. (E) Schematic of the laser experiment. Particles with $P>P^{*}$ are illuminated with a time-shared laser beam, which triggers their propulsion at velocity $v_{0} \hat{p}$ (25). 
to nonreciprocal interactions between individuals (i.e., $j \in V_{i}^{\alpha}$ does not imply $\left.i \in V_{j}^{\alpha}\right)(22,23)$. Consequently, the perception strongly depends on the orientation of the observer (Fig. 1, B and C). Indeed, the perception of an outward-pointing observer $\left(P^{\text {out }}\right)$ monotonically decays from the group center. However, the perception of an inward-pointing observer $\left(P^{\mathrm{in}}\right)$ exhibits a maximum at the edge of the group. The two perceptions coincide in the center, where they take the value

$$
P_{\alpha}^{\mathrm{c}}=\frac{\alpha}{\pi} \rho_{0} R_{0}
$$

which depends on the group number density $\rho_{0}$ and radius $R_{0}$ (25). The nonmonotonicity of $P^{\text {in }}$ implies that the perception of observers at the edge pointing inward is larger than that in the center of the group. This feature is absent for isotropic vision $(\alpha=\pi)$, where the perception $P^{\text {iso }}$ always decays monotonically, independently of the observer's direction (Fig. 1C).

In response to the visual perception, we make individuals vary their propulsion speed (i.e., their motility) according to the rule illustrated in Fig. 1D. When the perception is below a threshold $\left(P \leq P^{*}\right)$, the individual has a null velocity and is "passive." Assuming Brownian individuals, the dynamics in the passive state only consist of translational and rotational diffusion, which randomizes the orientation $\hat{p}$. Otherwise $(P>$ $\left.P^{*}\right)$, the individual propels at velocity $v_{0} \hat{p}$ and is "active." Trajectories in the active state are rather straight over a persistence length $l_{\mathrm{p}}=$ $v_{0} \tau_{R}$, where $\tau_{R}$ is the rotational diffusion time (26). Such a perception-response rule models a "social behavior" that encourages individuals to join crowded regions in their field of view and allows periods of passive search otherwise, resembling the intermittent motion of many living organisms (27).
The above behavior is experimentally realized using Janus particles of diameter $\sigma=4.28 \mu \mathrm{m}$, whose propulsion is triggered by light (28) and adjusted by a feedback loop (2I). Real-time image analysis yields the particles' positions and orientations, allowing us to allocate a vision cone to each particle and compute its perception using Eq. 1 (25). The individual motility response is realized by sending laser spots onto each particle (25) (Fig. 1E). When $P>P^{*}$, the particle is illuminated such that it propels with velocity $v_{0}=0.2 \mu \mathrm{m} / \mathrm{s}$, which is fixed throughout this work. Otherwise, the intensity is set to zero and the particle remains passive. Note that the rotational diffusion time $\tau_{\mathrm{R}}=107 \mathrm{~s}$ is unaffected by laser illumination (25) (fig. S1), which leads to $l_{\mathrm{p}}=21.4 \mu \mathrm{m}$. Particle positions and spot intensities are updated at $2 \mathrm{~Hz}$, ensuring quasi-static propulsion conditions (25). We thus obtain a sizable assembly of ABPs with tunable and nonreciprocal interactions.
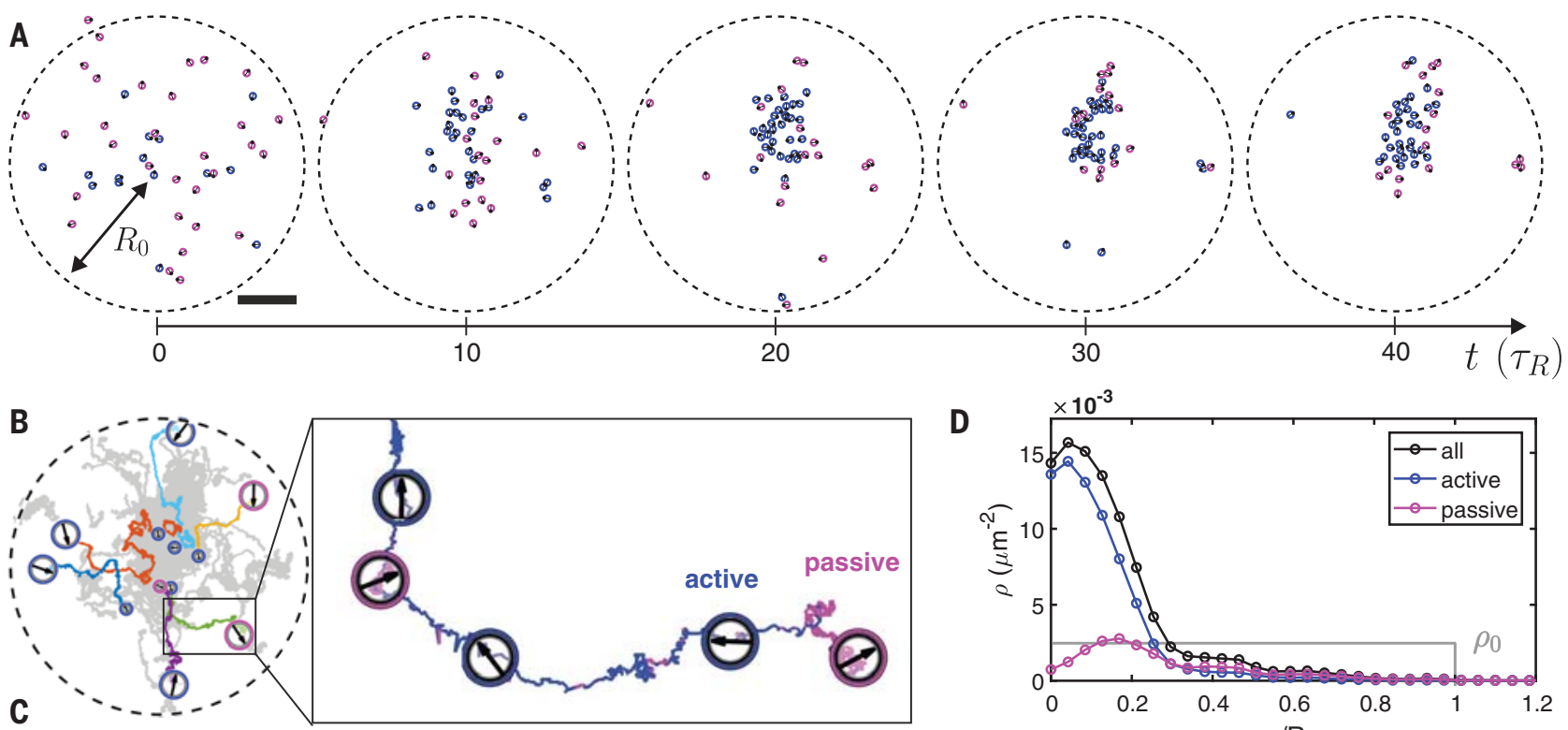

D
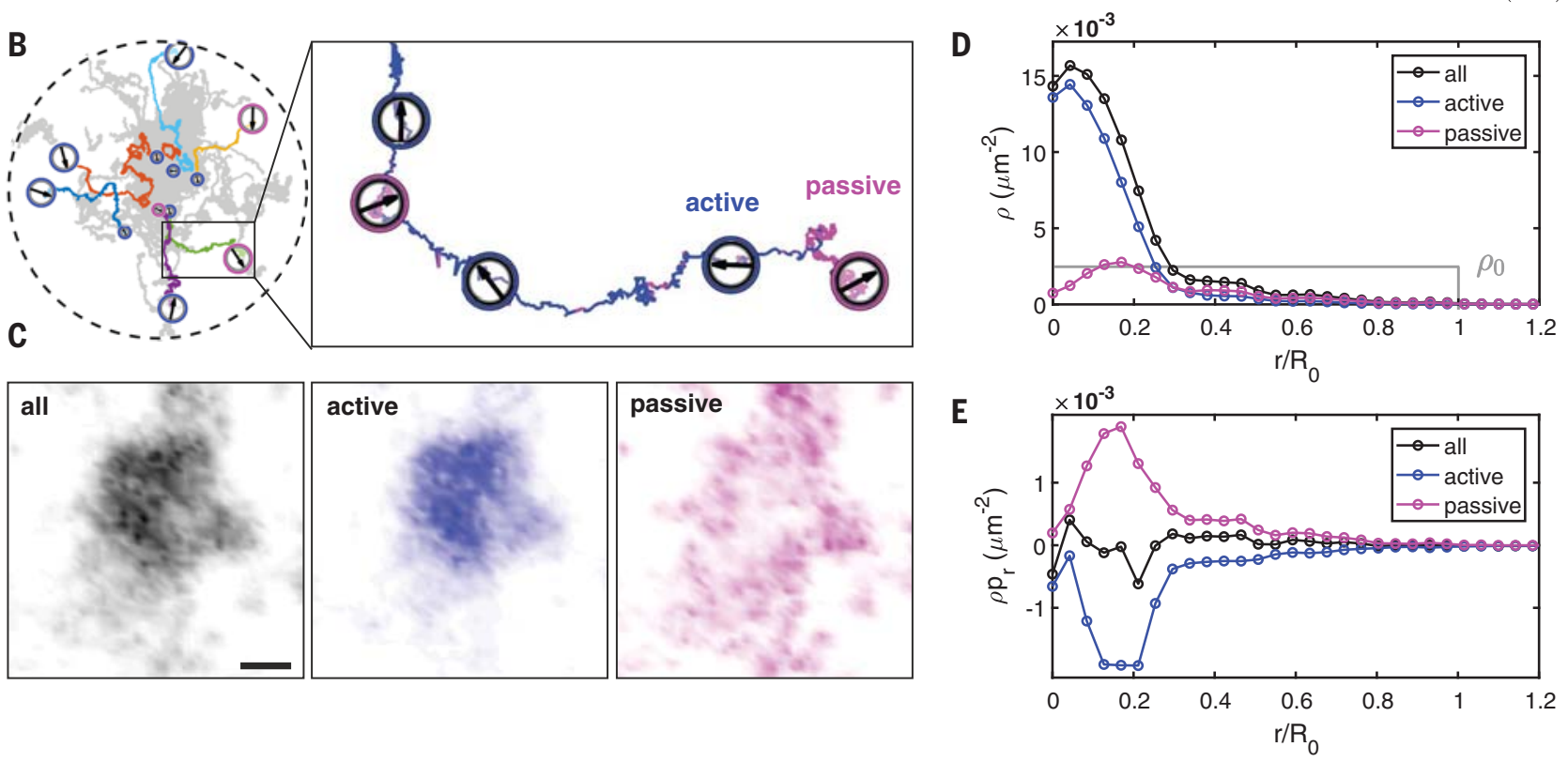

Fig. 2. Formation and structure of a cohesive active fluid.

(A) Formation of a cohesive group from a circular homogeneous distribution of active particles, in an experiment with $\alpha=\pi / 4$, $v_{0}=0.2 \mu \mathrm{m} / \mathrm{s}$, and $P^{*}=P_{\alpha}^{c}$. The dashed circles with radius $R_{0}$ correspond to the initial particle configuration and are fully permeable to particles. Blue and magenta correspond to the active and passive states, respectively. Scale bar, $40 \mu \mathrm{m}$. (B) Particle trajectories (gray) for the same run, with several highlighted trajectories (colors). Particles are shown at the starting point (large size) and at the end (smaller size) of each trajectory. The inset shows details of a trajectory and particle orientation at short times during several multiples of $\tau_{R}$. (C) Twodimensional maps of the particle density in the steady state for all, active, and passive particles. The color intensity is proportional to the density; see (D). Scale bar, $25 \mu \mathrm{m}$. (D) Radial density profile for all, active, and passive particles, together with the initial density profile $\rho_{0}$. (E) Same representation for the radial polarization density $\rho p_{r}$. 
We begin by examining the collective behavior under our perception-response rule for a vision cone with $\alpha=\pi / 4$. Experiments start from a homogeneous distribution of $N \approx 75$ particles within a circular region of radius $R_{0}=106 \mu \mathrm{m}$ $\left(\rho_{0}=2.1 \times 10^{-3} \mu \mathrm{m}^{-2}\right)$ with empty surroundings (Fig. 2A, dashed circles). We first consider the situation in which the perception threshold is set to $P^{*}=P_{\alpha}^{\mathrm{c}}=\alpha N /\left(\pi^{2} R_{0}\right)$ (Eq. 2). In this case, the particles start gathering into a smaller and denser group within $\sim 30 \tau_{\mathrm{R}}$ (Fig. $2 \mathrm{~A}$ and movie $\mathrm{S} 1)$. Afterward, a steady state is reached where no particles leave the group, indicating cohesion without active reorientations (25) (fig. S2). This contrasts with the case of permanently active particles, which would rapidly spread beyond $R_{0}$ by enhanced long-time diffusion (26).

To understand this transient process of group formation at a qualitative level, we discuss the behavior of a particle starting at the edge of the initial configuration and pointing outside (Fig. 2B). According to Fig. 1, B and C (case III), its perception is $P^{\text {out }}\left(R_{0}\right)=0$, so it is passive. After typically $\tau_{\mathrm{R}}$, the particle starts pointing toward the group, so the perception switches to $P^{\text {in }}\left(R_{0}\right)$, which is the maximum (see case II). This implies that $P^{\mathrm{in}}\left(R_{0}\right)>P_{\alpha}^{\mathrm{c}}$, and because the threshold $P^{*}$ is set to $P_{\alpha}^{\mathrm{c}}$, the particle is guaranteed to become active. The particle then moves toward the center, contributing to the formation of the group (Fig. 2B). On the other hand, the perception $P^{\text {out }}$ of particles moving away from the group rapidly decays below the threshold (Fig. 1C), which prevents them from leaving.

In the steady state, the particle density profile $\rho(r)$ decays to zero far from the core of the group (i.e., its surroundings are empty). In the center, $\rho$ is enhanced relative to the initial state with $\rho_{\text {core }} \approx 7 \rho_{0}$ (Fig. $2, \mathrm{C}$ and D), which corresponds to an area fraction of only $22 \%$, indicating that the group is fluid-like. This is in sharp contrast to close-packed clusters surrounded by a gas, as is usually observed (11-14). The cohesive active fluid observed here closely resembles the vivid aspect of natural swarms with no polar order $(24,29)$.

To understand the origin of cohesion, we computed the positional and orientational density distribution of active and passive particles, respectively. As shown in Fig. 2, C and D, active particles are dominantly located at the core of the group, whereas passive particles are dominantly located at the edge of the group. In Fig. $2 \mathrm{E}$ we show the radial polarization density, $\rho p_{\mathrm{r}}$, where $p_{\mathrm{r}}=\langle\hat{p} \cdot \hat{r}\rangle$ is the average radial component of the particle orientation $\hat{p}$. Clearly, the radial polarization density of passive particles is positive, meaning that they point outward. The opposite is true for active particles. Note that the overall polarization is zero. This correlation between particle position and polarization shows that escape events $\left(\rho p_{\mathrm{r}}>0\right)$ are penalized by loss of activity, whereas joining the group $\left(\rho p_{\mathrm{r}}<0\right)$ is promoted by activity. Thus, the cohesion mechanism is purely induced by motility and not by attractive pair interactions or torques. Note that the activity switches that depend on the orientation at the group edge in our overdamped system are analogous to the centripetal acceleration that ensures the cohesion of insect swarms (30).

To go beyond this simple picture of the cohesion mechanism, we gain a more rigorous insight on the processes at stake by performing simulations with point-like ideal gas particles and using analytical arguments (25). Ideal gas particles following the same perception-response rule also form a cohesive active fluid (movie S2), confirming the generic nature of such a cohesion mechanism. In fact, one can show that group formation arises not only for a sharp threshold response (Fig. 1D), but generally when the propulsion velocity is an increasing function of a long-range and anisotropic perception (25). We find that a linear instability effectively leads particles to slow down when oriented against density gradients, which impedes them from leaving the group when they point outward (fig. S3). We derive this instability in the limit $\alpha \rightarrow 0$, where interactions are highly nonreciprocal (25). Thus, this motility-induced cohesion is purely due to nonreciprocity and not to alignment mechanisms, as in previous studies $(22,23)$.

We evaluated the impact of vision on group formation by varying $\alpha$ in the full range $[0 ; \pi]$, still using $P^{*}=P_{\alpha}^{\mathrm{c}}$. Both experiments and simulations show that at low $\alpha$, groups tend to have an elongated shape with large shape fluctuations, whereas they become more circular and dilute when $\alpha$ increases toward $\pi / 2$ (Fig. $3 \mathrm{~A}$ and movies S1 to S5). When $\alpha>\pi / 2$, group formation is no longer observed (Fig. 3A). To quantify this breakdown of group formation, we determined the mean number of particles $\langle s\rangle$ per aggregate as a function of $\alpha$ [two particles are considered in the same aggregate when closer than the persistence length $\left.l_{\mathrm{p}}(25)\right]$ (Fig. 3B). At low $\alpha,\langle s\rangle / N \approx 0.8$, showing that virtually all particles belong to the same aggregate (i.e., they form a single cohesive group). However, at high $\alpha,\langle s\rangle / N$ sharply decreases, indicating the loss of the single group in favor of small aggregates. Such overall dependence
Fig. 3. Breakdown of group formation and definition of response horizon. (A) Density maps for various vision cone angles $\alpha \in[0 ; \pi]$. Group formation breaks down for $\alpha>\pi / 2$ (right). Scale bar, $50 \mu \mathrm{m}$. (B) Mean size of aggregates relative to the number of particles $\langle s\rangle / N$ as a function of $\alpha$ for experiments (red circles) and simulations (red line), together with the fraction of particles beyond the response horizon $\phi_{h}$ for experiments (black circles) and simulations (black line). The thick and thin dashed vertical lines correspond to the angle where $h=R_{0}$, as obtained numerically and in the far field, respectively; see (D). Error bars denote SD. (C) Radial perception profiles for three values of $\alpha$ and definition of the response horizon $h$ (blue dashed lines) in the case $P^{*}=P_{\alpha}^{c}$ (horizontal black line). (D) Response horizon obtained numerically and in the far-field approximation. (E) Schematic of the location of $h$ for low $\alpha$ and $P^{*}=P^{c}$ (F) Same for high $\alpha$.
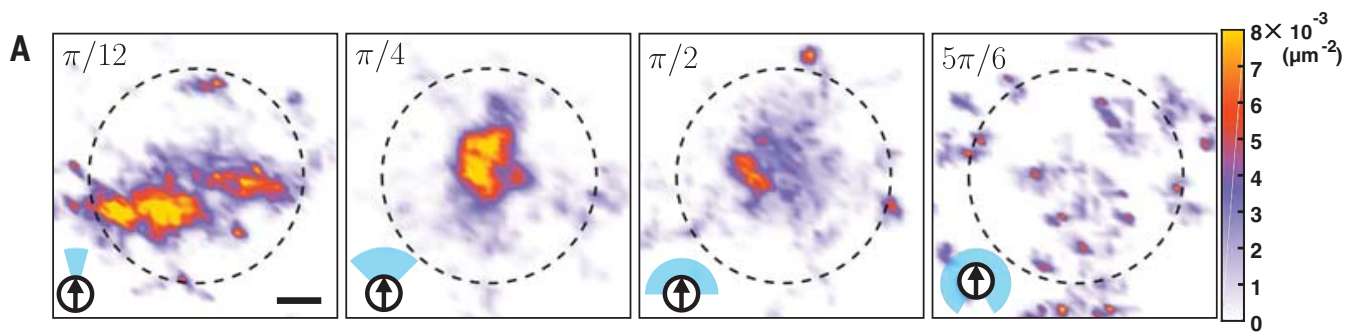

B

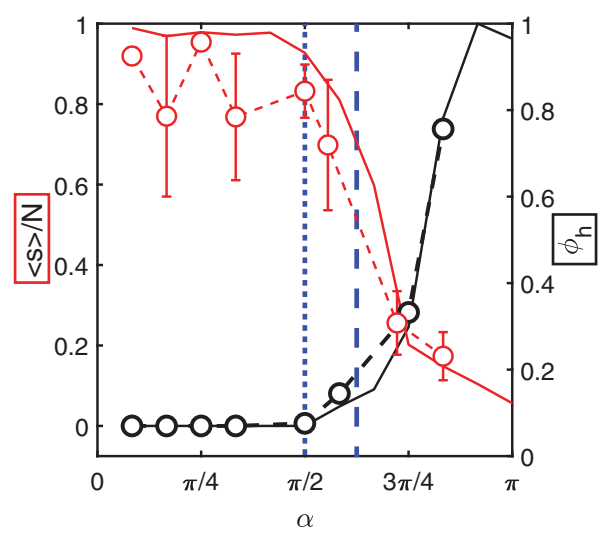

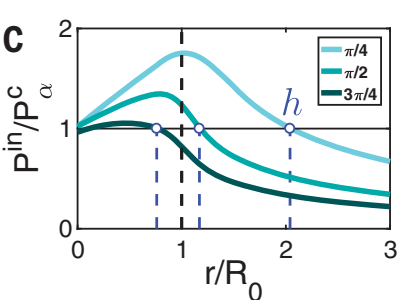

D

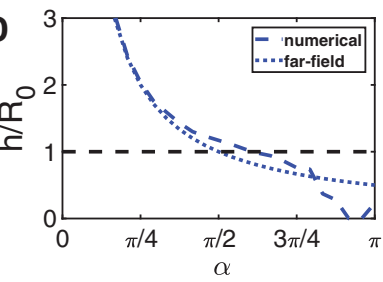

E

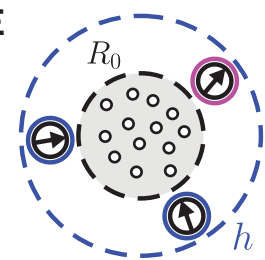

F

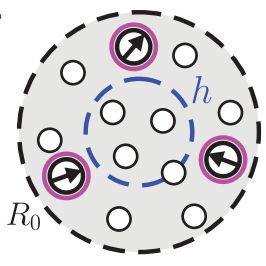


is in good agreement with our simulations of point-like particles.

Group formation breaks down when the perception of particles being too far from the group center never exceeds the threshold, regardless of their orientation. Accordingly, beyond a distance $h$, the "response horizon," particles are always passive. Because particles pointing toward the group always have the largest perception $P^{\text {in }}$, the response horizon is defined by $P^{\text {in }}(h)=P^{*}$ (Fig. 3C). A numerical calculation of $P^{\text {in }}$ from Eq. 1 with $P^{*}=P_{\alpha}^{\mathrm{c}}$ yields $h$ for different values of $\alpha$ (25). Figure 3D shows that $h$ is then a decreasing function of $\alpha$. Indeed, in the far field, the perception approximates to $P^{\text {in }}(r) \approx N /(2 \pi r)$ (see Eq. 1). Taking $P^{\mathrm{in}}(h)=P^{*}=P_{\alpha}^{\mathrm{c}}$ and using Eq. 2 yields $h=\pi R_{0} /(2 \alpha)$, which decreases upon increasing $\alpha$ (Fig. 3D). Thus, at low $\alpha, h \gg R_{0}$, which prevents particles in the initial configuration from being irreversibly passive (Fig. 3E). However, at larger $\alpha, h$ becomes smaller than $R_{0}$. Thus, particles located at distances $h<r<$ $R_{0}$ remain passive, irrespective of their orientation, and may not join the group (Fig. 3F). The loss of group formation should then occur when $h=R_{0}$. To test this idea, we determined the fraction $\phi_{h}$ of particles located beyond $h$ for each value of $\alpha$ (Fig. 3B). We found that $\phi_{h} \approx 0$ at low $\alpha$ but increases sharply for high $\alpha$; notably, $\phi_{h}$ starts being nonzero at $\alpha=\pi / 2$, consistently at the point where $\langle s\rangle / N$ starts collapsing in Fig. 3B. This means that the loss of group formation is indeed due to particles located outside the response horizon. This threshold value of $\alpha=\pi / 2$, which we observed in both experiments and simulations, is slightly smaller than suggested by the condition $h=R_{0}$ (Fig. 3D). Still, the value $\pi / 2$ can be obtained from the far-field expression $h=\pi R_{0} /(2 \alpha)$, which is equal to $R_{0}$ when $\alpha=\pi / 2$.

We now remove the constraint $P^{*}=P_{\alpha}^{\mathrm{c}}$ and report the results of experiments for different threshold values in a vision threshold diagram $\left(\alpha, P^{*}\right)$, where $P^{*}$ is normalized by $P_{\alpha}^{\mathrm{c}}(25)$ (Fig. 4). Clearly, cohesive groups are always obtained for $P^{*} \leq P_{\alpha}^{\mathrm{c}}$ and $\alpha \leq \pi / 2$. Because $h>R_{0}$ is guaranteed in these cases, the group forms and becomes stable with a high fraction of active particles (Fig. 4 , case I). Interestingly, group formation is possible even for $\alpha>\pi / 2$, provided that $P^{*}$ is sufficiently low relative to $P_{\alpha}^{\mathrm{c}}$ (i.e., individuals with wide fields of view can form a group by lowering their threshold). From simulations, the domain boundary of group formation is determined as where $\phi_{h}$ becomes nonzero $\left(\phi_{h}=0^{+}\right)$and is in remarkably good agreement with the experimental points. Beyond this limit, group formation can be lost in two ways. First, when $h<R_{0}$ in the initial configuration (star symbols), this causes particles initially beyond $h$ to be irreversibly passive and group formation fails (most particles eventually become passive, case II). This domain typically corresponds to high $\alpha$ and $P^{*} / P_{\alpha}^{\mathrm{c}}$, and is indeed well delimited by the line $R_{0}=h$ (Fig. 4). Second, between the $\phi_{h}=0^{+}$and $R_{0}=h$ boundaries, $h>R_{0}$ and most particles are active in the initial configuration (triangles). At such high $\alpha$, however, $P^{\text {in }} \approx P^{\text {out }}$, akin to the iso-
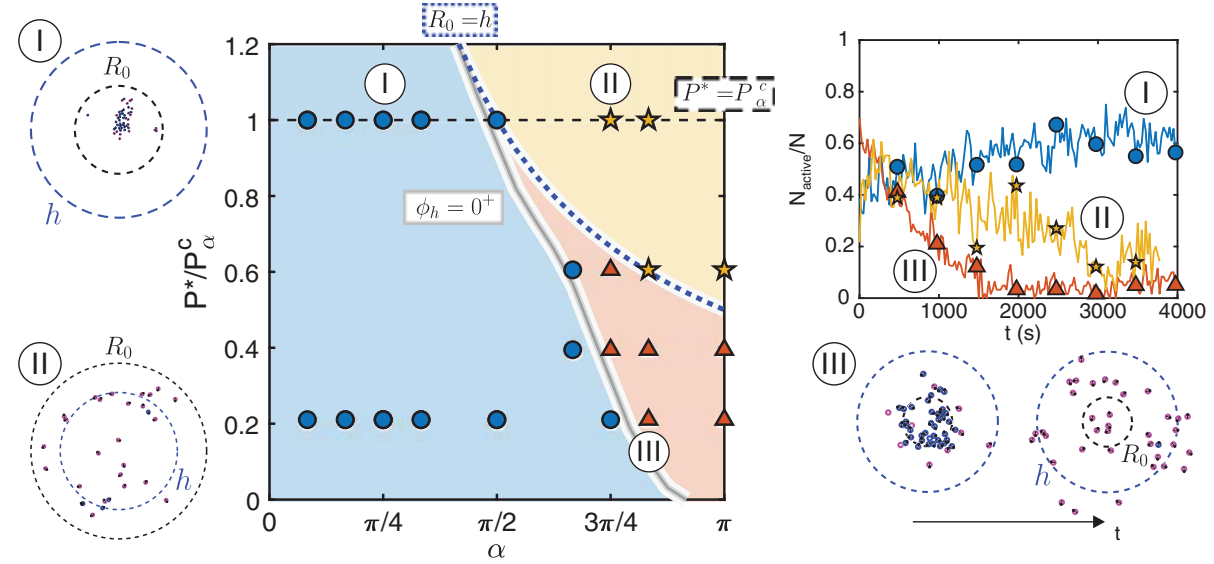

Fig. 4. Vision threshold diagram. Experimental points (symbols) represent different values of the response threshold $P^{*}$ and vision cone angle $\alpha$. Three domains are distinguished: group formation and cohesion (circles, case I), no formation (stars, case II), and no cohesion (triangles, case III). The fraction of active particles $N_{\text {active }} / N$ as a function of time is shown for the three cases (top right), together with snapshots (not to scale) at early time (case III) and late time (cases I, II, and III). The domain boundaries correspond to when $\phi_{h}$ starts being nonzero in the simulations $\left(\phi_{h}=0^{+}\right.$, gray line) and $R_{0}=h$ in the far field (blue dashed line). The cases $P^{*}=0$ and $\alpha=0$ lead all particles to be active and passive, respectively (not shown)

tropic case (see Fig. 1C), so cohesion is lost and the group expands until particles cross $h$, becoming all passive (case III).

We did complementary experiments (25) to see how inserting a blind zone between the two halves of the vision cone affects cohesion (fig. S4). For a narrow blind zone, vision is mainly frontal and cohesion is maintained, as in Fig. 4, case I. Conversely, for a wide blind zone, vision is mainly lateral and cohesion is lost, as in Fig. 4, case III. This is because for frontal vision, the direction of perception is aligned with that of motion, which enables the particle to join crowded regions. Conversely, for lateral vision, the direction of perception is orthogonal to that of motion, which prevents the particle from joining crowded regions. As such, the presence of a front-rear asymmetry in the vision along the direction of motion is crucial for the emergence of cohesion.

We note that the outcome of our perceptionresponse rule can be understood in light of the observed link between vision and foraging modes of animals in nature (31). The narrow vision of predators (31) is an advantage in reaching target groups because motion toward the group can be triggered even far away $\left(h \gg R_{0}\right.$ ) for perception thresholds typically up to $P_{\alpha}^{\mathrm{c}}$ (Fig. 4 , case I). Conversely, prey animals usually have wide fields of view (31), which is an obstacle to forming groups ( $h<R_{0}$ when $P^{*} \approx P_{\alpha}^{\text {c }}$ Fig. 4 , case II) unless they compensate by staying alert (i.e., by setting their reaction threshold low enough). Similarly, the effects of visual occlusion can be corrected by lowering the threshold to compensate for the fewer individuals in sight. Note that setting their threshold relative to the background level, $P_{\alpha}^{\mathrm{c}}$, does not require prey or predators to know the exact number of individuals in their field of view, but only to estimate the group density and size according to Eq. 2.
This motility-induced cohesion fulfills two important requirements in robotics applications: It is both robust and scalable. The robustness to noise and imperfections of a real system is a known feature of group behavior based on instability mechanisms (32), such as the one proposed here. The scalability comes from the fact that the zone of cohesion (Fig. 4, case I) depends only on the value of the threshold relative to $P_{\alpha}^{\mathrm{c}}$, regardless of the absolute value of the group size and density. This provides a potential solution to aggregating arbitrary numbers of robots in the absence of centralized control $(32,33)$.

We have shown that robust group formation and cohesion can be obtained by a simple increase of the motility as a function of an anisotropic and long-range perception of the environment, without the need for active reorientation. We have obtained cohesive active fluids of responsive active particles in an experimental system and confirmed the generic character of this mechanism in simulations of point-like particles. We find that this perception-response rule results in an effective response of the particles to density gradients, akin to the behavior of larvae with gradient sensing (34). We hope to stimulate future theoretical studies in order to provide a full description of this instability within the framework of motility-induced phenomena. In general, narrow fields of view and/or low thresholds of response facilitate group formation in a robust and scalable manner. Our results point toward the importance of anisotropic and long-range sensing-achieved, for example, by directed vision or an asymmetrical distribution of receptors on the body-as a general factor in the emergence of cohesion when individuals are subject to internal reorientation time scales that remain unchanged by the presence of others. Our work also illustrates 
the relevance of testing simple algorithms to obtain robust and scalable behavior of microrobots with limited sensing and response capabilities. We thus foresee that soft matter systems with individual control of the particles represent an interesting class of tabletop experiments with inherent sources of noise, and that such experiments can lead to quick, low-priced, and versatile prototyping of procedures relevant to the collective behavior of biological, social, and robotic systems.

\section{REFERENCES AND NOTES}

1. M. Ballerini et al., Proc. Natl. Acad. Sci. U.S.A. 105, 1232-1237 (2008).

2. A. Berdahl, C. J. Torney, C. C. Ioannou, J. J. Faria, I. D. Couzin, Science 339, 574-576 (2013).

3. J. L. Silverberg, M. Bierbaum, J. P. Sethna, I. Cohen, Phys. Rev. Lett. 110, 228701 (2013).

4. D. J. G. Pearce, A. M. Miller, G. Rowlands, M. S. Turner, Proc. Natl. Acad. Sci. U.S.A. 111, 10422-10426 (2014).

5. D. Gorbonos et al., New J. Phys. 18, 073042 (2016).

6. R. R. Kay, P. Langridge, D. Traynor, O. Hoeller, Nat. Rev. Mol. Cell Biol. 9, 455-463 (2008)

7. I. D. Couzin, J. Krause, R. James, G. D. Ruxton, N. R. Franks, J. Theor. Biol. 218, 1-11 (2002).

8. K. Tunstrøm et al., PLOS Comput. Biol. 9, e1002915 (2013).

9. A. Cavagna, I. Giardina, Annu. Rev. Condens. Matter Phys. $\mathbf{5}$, 183-207 (2014).
10. P. Romanczuk, M. Bär, W. Ebeling, B. Lindner, L. Schimansky-Geier, Eur. Phys. J. Spec. Top. 202, 1-162 (2012).

11. I. Theurkauff, C. Cottin-Bizonne, J. Palacci, C. Ybert, L. Bocquet, Phys. Rev. Lett. 108, 268303 (2012).

12. J. Palacci, S. Sacanna, A. P. Steinberg, D. J. Pine, P. M. Chaikin, Science 339, 936-940 (2013).

13. F. Ginot, I. Theurkauff, F. Detcheverry, C. Ybert, C. Cottin-Bizonne, Nat. Commun. 9, 696 (2018)

14. I. Buttinoni et al., Phys. Rev. Lett. 110, 238301 (2013).

15. M. E. Cates, J. Tailleur, Annu. Rev. Condens. Matter Phys. 6, 219-244 (2015).

16. T. Vicsek, A. Czirók, E. Ben-Jacob, I. Cohen, O. Shochet, Phys. Rev. Lett. 75, 1226-1229 (1995).

17. A. Bricard, J.-B. Caussin, N. Desreumaux, O. Dauchot, D. Bartolo, Nature 503, 95-98 (2013)

18. G. Grégoire, H. Chaté, Phys. Rev. Lett. 92, 025702 (2004).

19. D. J. G. Pearce, M. S. Turner, New J. Phys. 16, 082002 (2014)

20. R. van Drongelen, A. Pal, C. P. Goodrich, T. Idema, Phys. Rev. E 91, 032706 (2015).

21. T. Bäuerle, A. Fischer, T. Speck, C. Bechinger, Nat. Commun. 9 , 3232 (2018).

22. L. Barberis, F. Peruani, Phys. Rev. Lett. 117, 248001 (2016)

23. M. Durve, A. Saha, A. Sayeed, Eur. Phys. J. E 41, 49 (2018).

24. A. Attanasi et al., PLOS Comput. Biol. 10, e1003697 (2014).

25. See supplementary materials.

26. J. R. Howse et al., Phys. Rev. Lett. 99, 048102 (2007).

27. J. J. Gibson, Br. J. Psychol. 49, 182-194 (1958).

28. J. R. Gomez-Solano et al., Sci. Rep. 7, 14891 (2017).

29. A. Cavagna et al., Nat. Phys. 13, 914-918 (2017).

30. D. H. Kelley, N. T. Ouellette, Sci. Rep. 3, 1073 (2013).
31. M. S. Banks, W. W. Sprague, J. Schmoll, J. A. Q. Parnell, G. D. Love, Sci. Adv. 1, e1500391 (2015).

32. I. Slavkov et al., Sci. Robot. 3, eaau9178 (2018).

33. L. Bayındır, Neurocomputing 172, 292-321 (2016)

34. J.-L. Deneubourg, J.-C. Grégoire, E. Le Fort, J. Insect Behav. 3 169-182 (1990).

\section{ACKNOWLEDGMENTS}

We thank D. Bartolo for useful discussions and C. Lozano for critically reading the manuscript. Funding: Supported by ERC Advanced Grant 693683 and the DFG Centre of Excellence 2117 "Centre for the Advanced Study of Collective Behaviour," ID: 422037984 (C.B.). Author contributions: C.B. and F.A.L. designed the research; T.B. developed the experimental setup; F.A.L. and H.W. acquired and analyzed the data; F.A.L. performed simulations and theory; F.A.L. and H.W. wrote the supplementary materials; C.B., F.A.L., and H.W. wrote the paper; and all authors commented on the manuscript. Competing interests: We declare no competing interests. 